\title{
Supplement to: The simultaneous occurrence of surge and discharge extremes for the Rhine delta
}
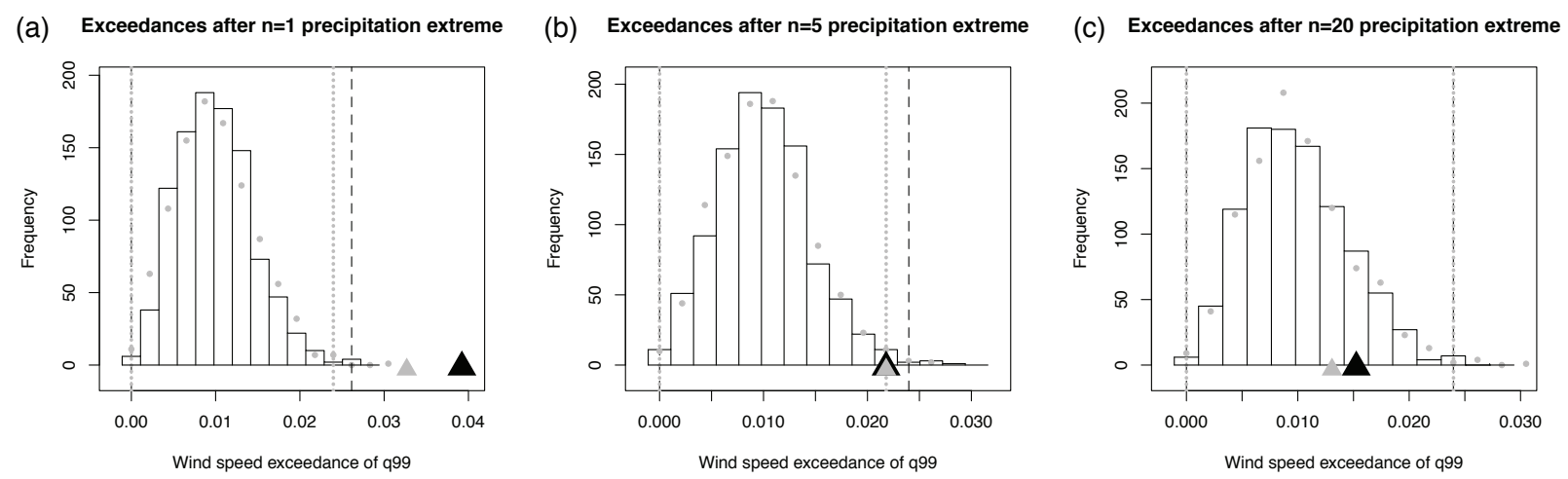

Figure S1: Exceedance of the climatological $q_{0.99}^{w}$ for the wind speed following extreme $n$-day precipitation sums (black triangle for current climate, grey for future) and 1000 random samples of the same size (black bars for current climate, dots for future). The vertical dashed lines enclose $99 \%$ of the 1000 samples (black for current climate, grey for future). 
(a)

Wind after $n=1$ precipitation extreme

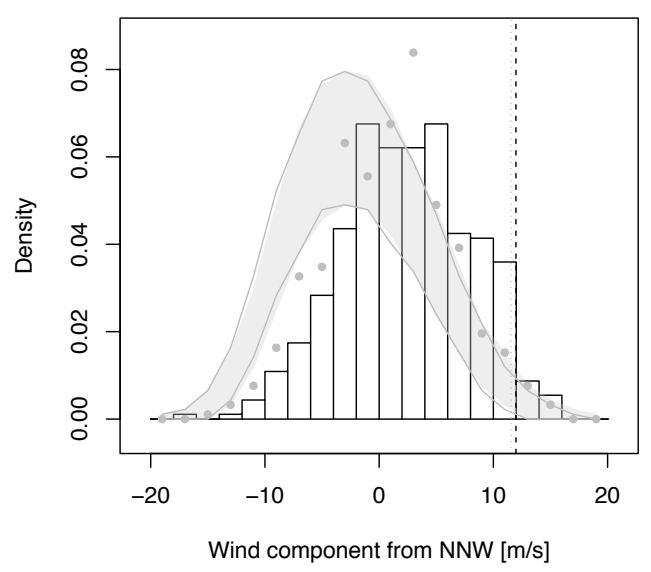

(c)

Wind after $n=5$ precipitation extreme

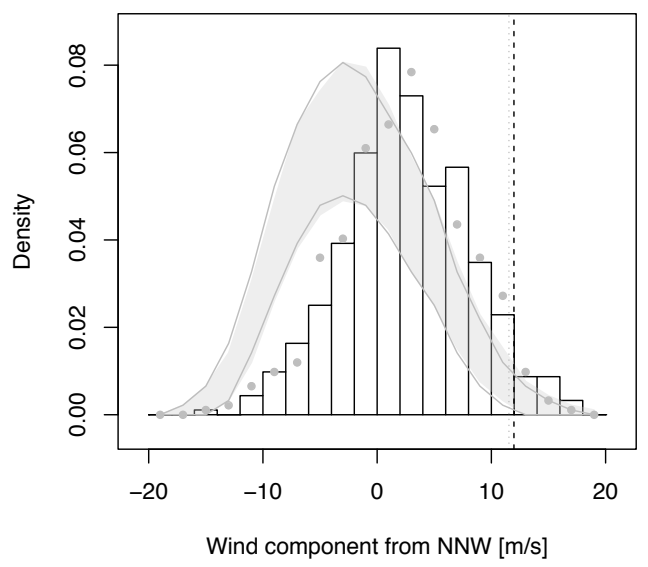

(e) Wind after $\mathrm{n}=\mathbf{2 0}$ precipitation extreme

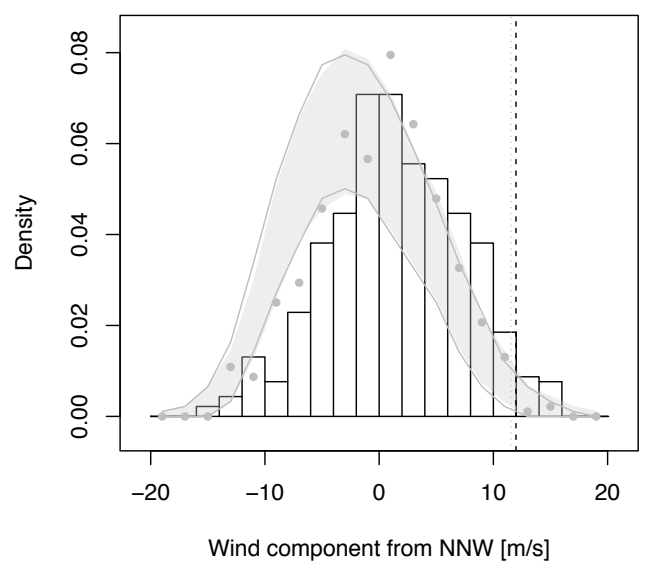

(b) Anomaly after $n=1$ precipitation extreme

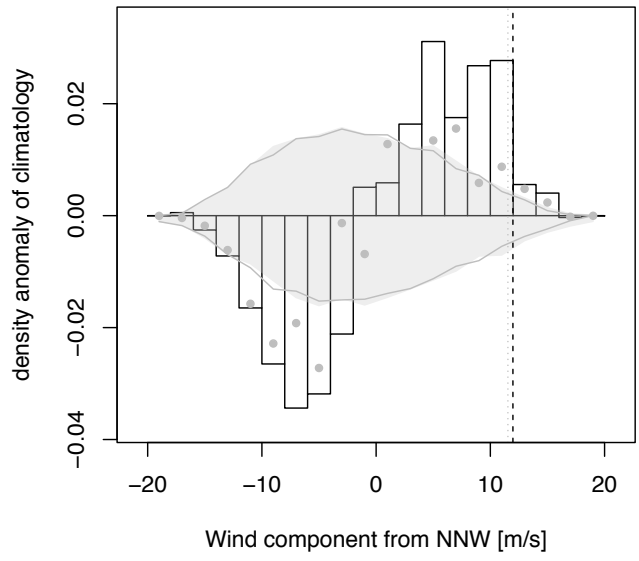

(d) Anomaly after $\mathrm{n}=5$ precipitation extreme

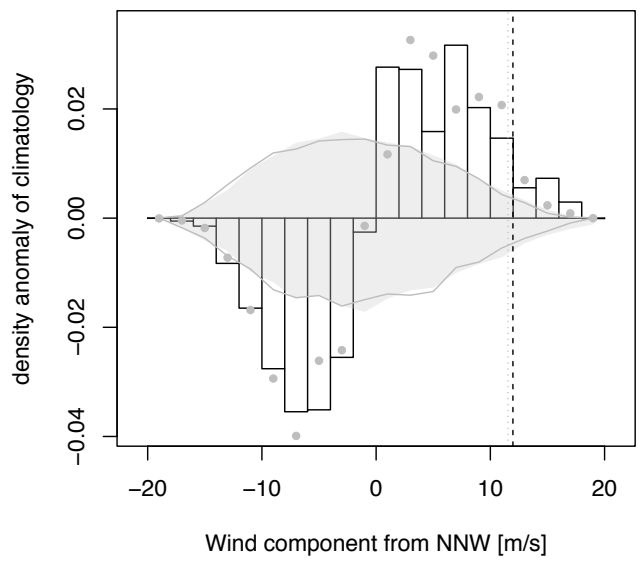

(f) Anomaly after $\mathrm{n}=20$ precipitation extreme

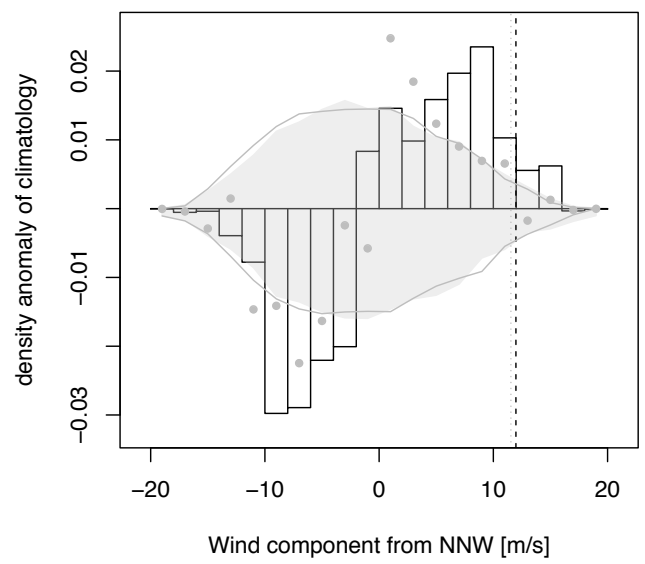

Figure S2: NNW wind component following extreme (bars for current climate 1950-1980, dots for future 2070-2100) and random samples (95\% density range, shaded for current climate, outlined for future) of $n$-day precipitation sums (left) and their anomaly with reference to climatology (right). The $q_{0.99}^{w}$ threshold is marked with a dashed line (black for current climate, grey for future). 

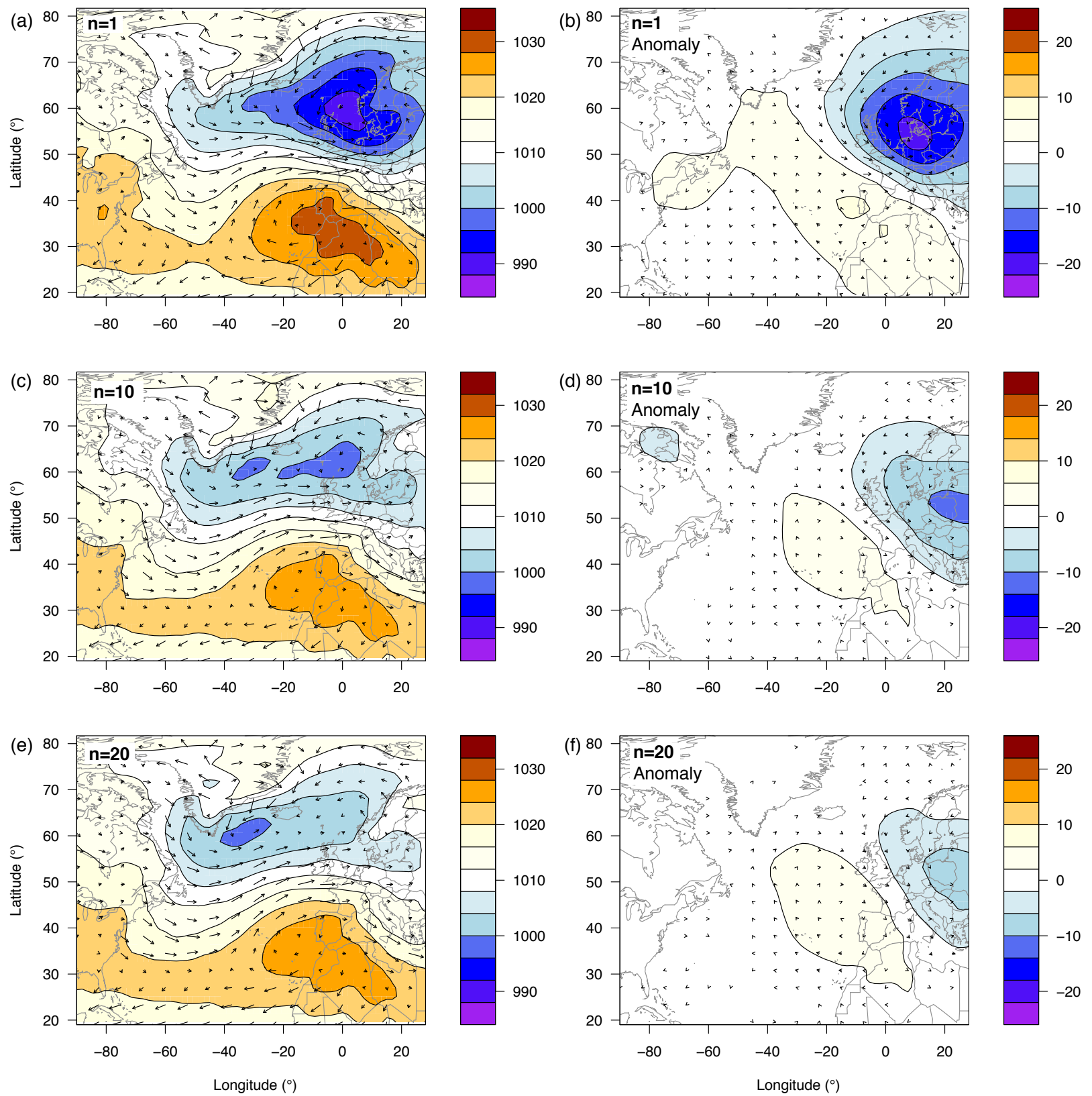

Figure S3: Precipitation conditioning: SLP climatology in hPa conditioned on 1-day (a-b), 10-day (c-d) and 20-day (e-f) precipitation extremes, for which the rainfall has fallen over the $n$-days preceding the synoptic situation shown. Each panel contains 459 entries. Left hand column contains conditional SLP climatologies, right hand column contains the anomaly of the conditional climatology with respect to the full climatology in Fig. 6a). 

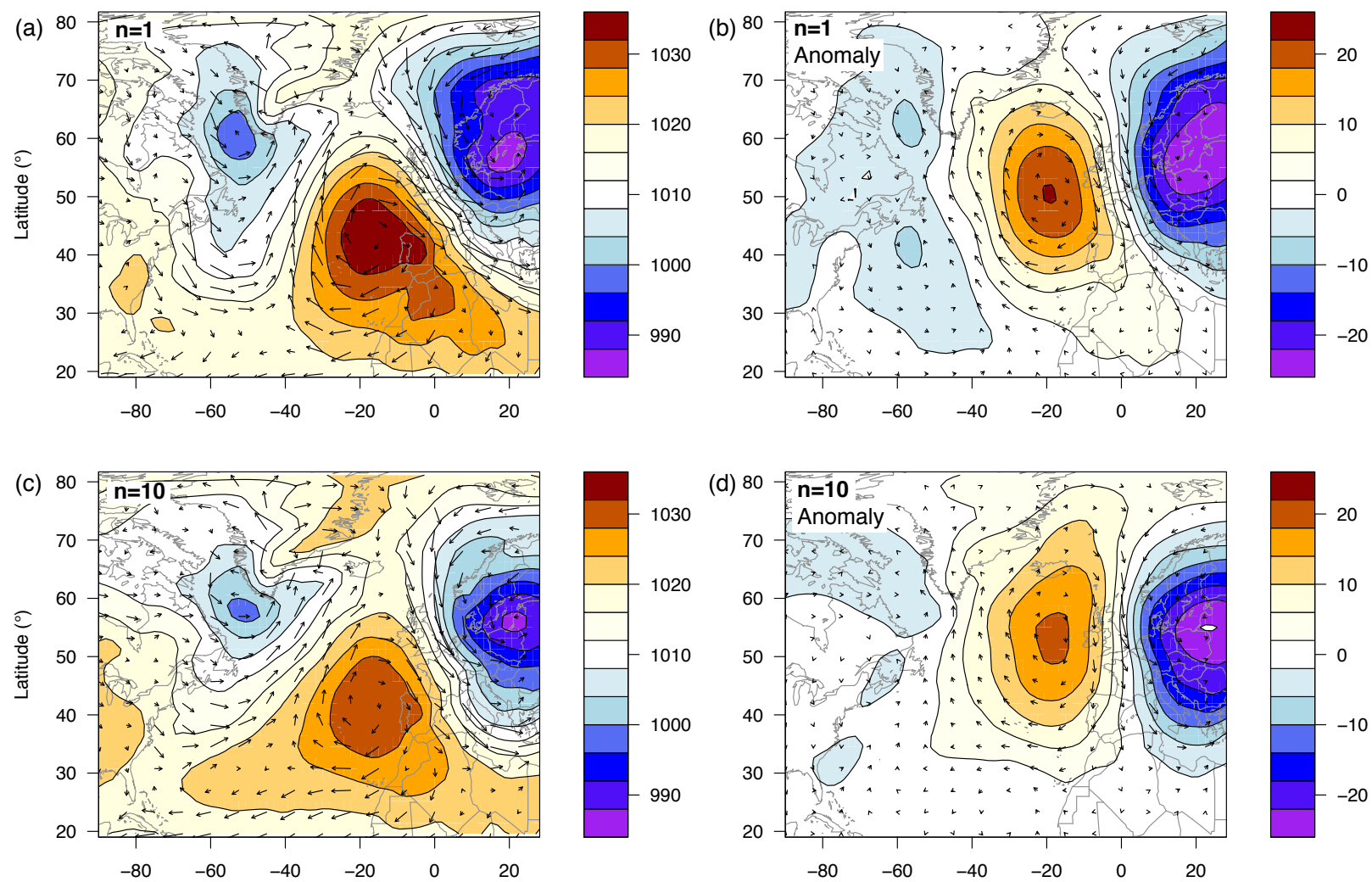

(d)
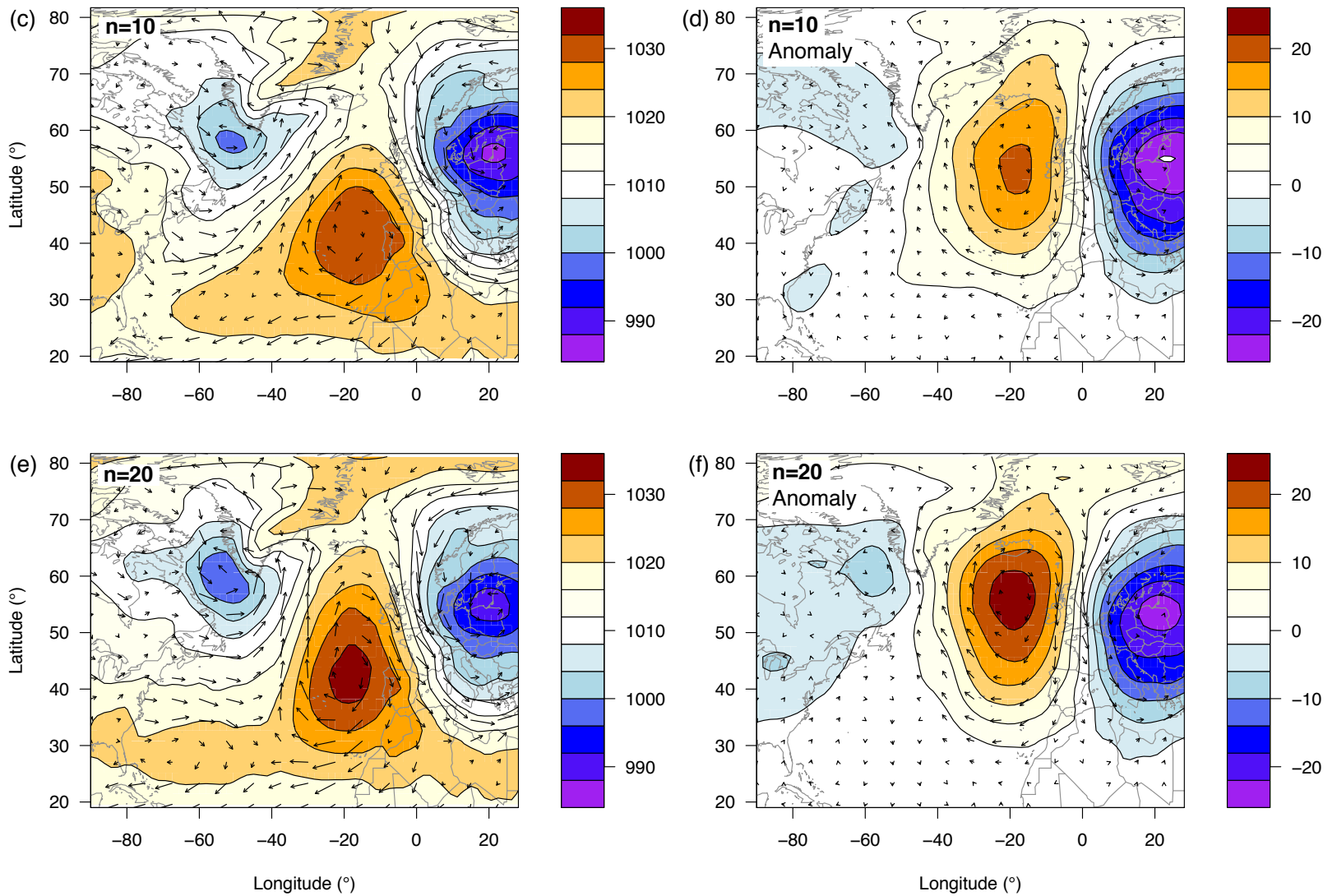

Figure S4: Joint event conditioning: SLP composite in $\mathrm{hPa}$ for events satisfying both wind and precipitation extremes following 1-day (a-b), 10-day (c-d) and 20-day (e-f) precipitation sums. Each panel contains on the order of 20 entries. Left hand column contains conditional SLP climatologies, right hand column contains the anomaly of the conditional climatology with respect to the full climatology in Fig. 6a). 

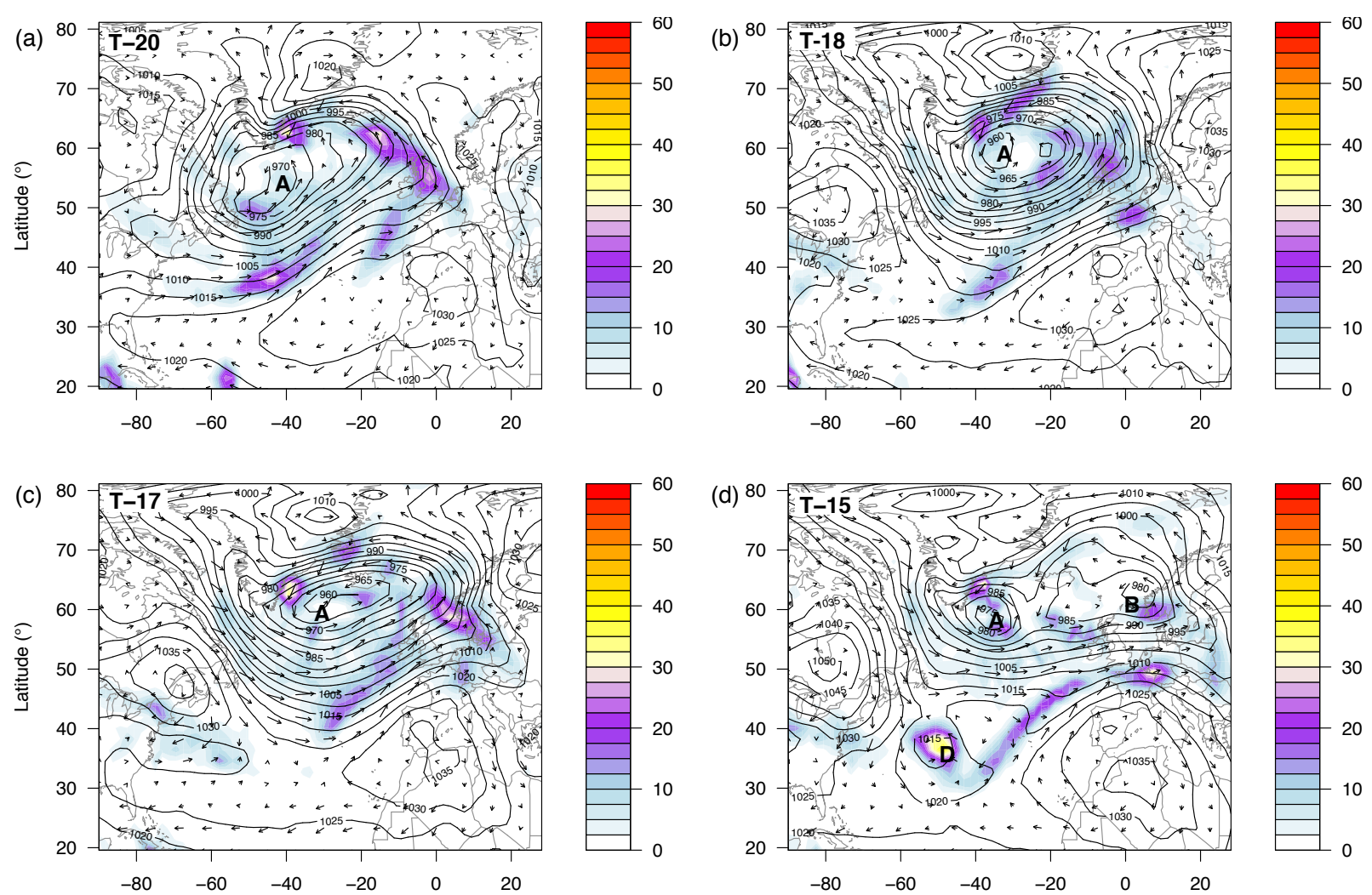

(d)
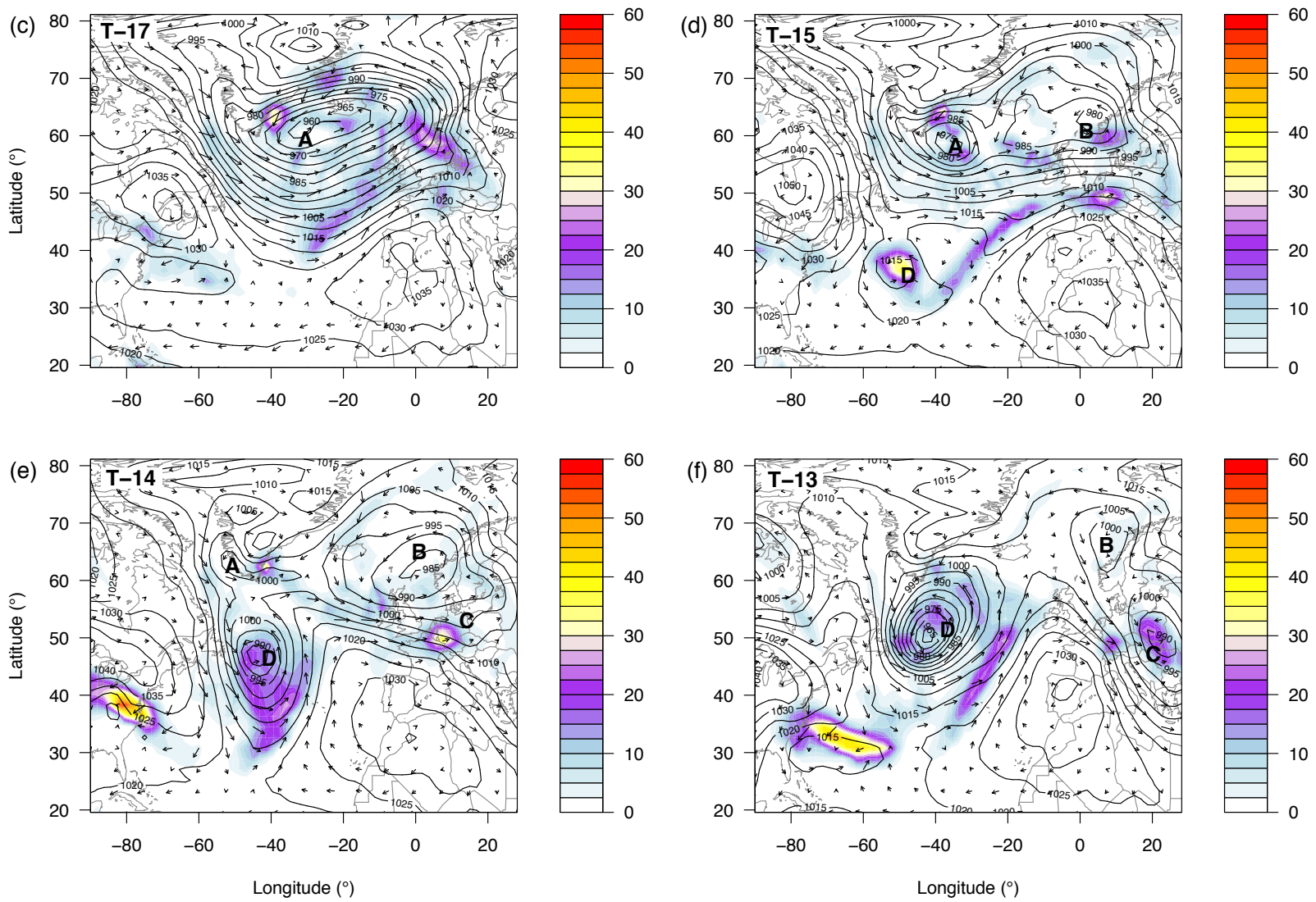

Figure S5: Evolution of synoptic situation for a single event that satisfied the joint extreme condition $(n=20$ for precipitation) at times $T$. Time references are in days relative to $T$. The SLP field (12 UTC) in $\mathrm{hPa}$ is contoured, precipitation in $\mathrm{mm} / 24 \mathrm{~h}$ is shaded, daily average wind vectors are superimposed. 

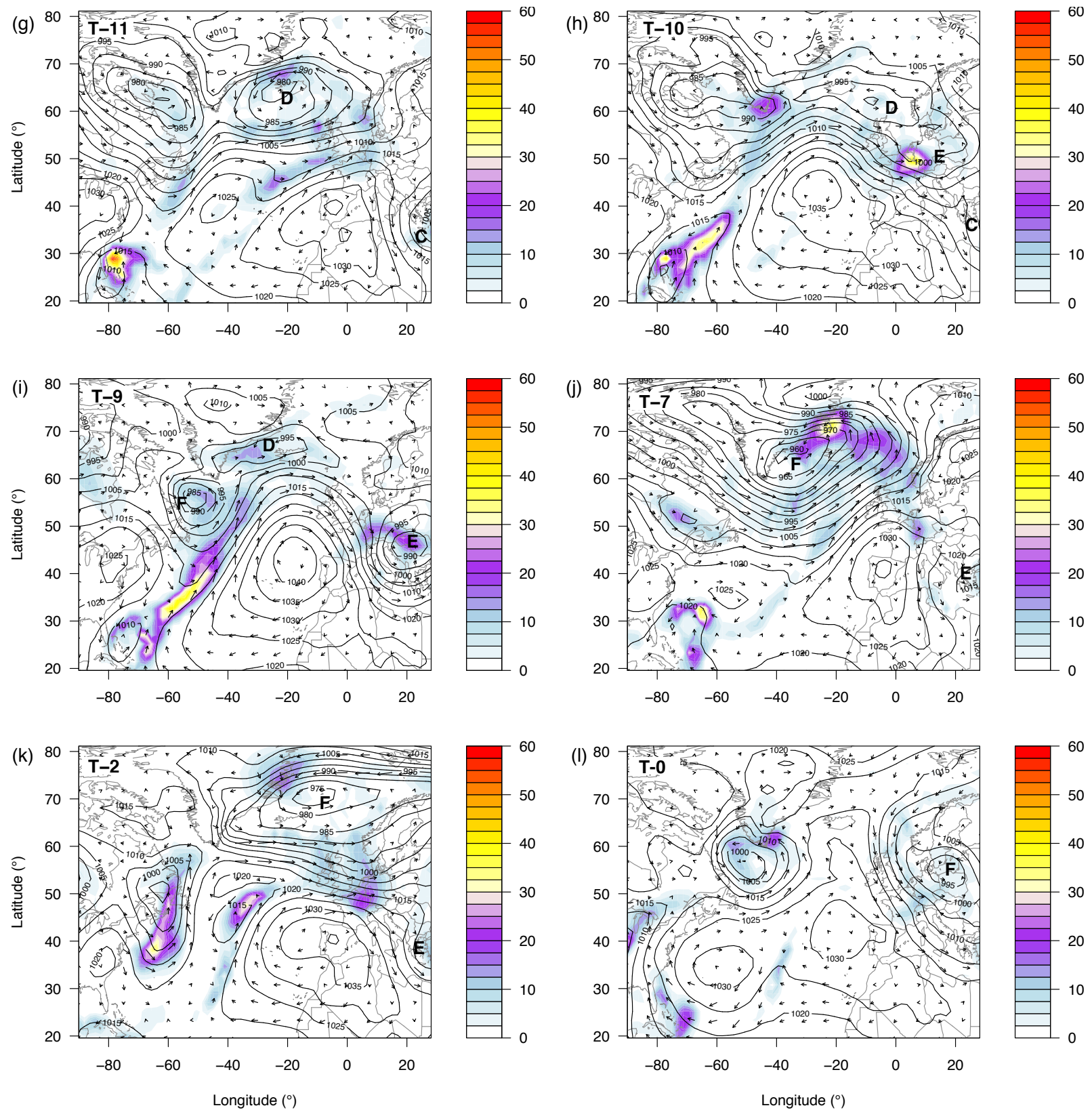

Figure S5: continued 